\title{
ON THE NUMBER OF REAL SOLUTIONS OF A RANDOM POLYNOMIAL
}

\author{
Submitted for publication to \\ J. Mathematical Analysis and Applications \\ K. FARAHMAND \\ University of Ulster, Department of Mathematics \\ Jordanstown, Co. Antrim BT37 0QB, UK \\ E-mail: k.farahmand@ulst.ac.uk
}

Let $(\Omega, A, \operatorname{Pr})$ be a fixed probability space and $a_{j}(\omega) ; j=0,1,2, \ldots, n$ be a sequence of independent random variables defined on $\Omega$ and

$$
P(x) \equiv P_{n}(x, \omega)=\sum_{j=0}^{n} a_{j}(\omega) x^{j}
$$

Assuming standard normal distribution for the coefficients $a_{j}(\omega) \equiv a_{j} ; j=0,1,2, \ldots$, $n$, it is known that the expected number of real zeros of $P(x)$ is asymptotic to $(2 / \pi) \log n$ as $n$ tends to infinity. The expected number of real solutions of the equation $P_{n}(x)=K$ or $K$-level crossings of $P_{n}(x)$, where $K$ ranges through a sequence $K_{n}$ such that $K_{n}^{2} / n \rightarrow 0$ as $n \rightarrow \infty$, is also known.

The objective of this paper is to generalize the known results in two directions. We relax the normality assumption for the coefficients by considering a general distribution with finite or infinite variance, and obtain the result for the number of solutions of $P(x)=K_{n}$, rather than its expected number. The trade off is to obtain bounds (fairly narrow) for this number rather than the asymptotic value derived for the expected number of solutions in the normal case.

Denote any positive absolute constant value, not necessarily the same at each occurrence, by $C$. We summarize our results in the following four theorems.

Theorem 1: Let $\sigma_{j}^{2}$ be the variance and $\tau_{j}^{3}$ be the third absolute moment of $a_{j}$ and

$$
\kappa_{n}=\max _{0 \leq j \leq n} \sigma_{j}, \quad t_{n} \overline{\overline{0} \leq j \leq n} \min _{j} \sigma_{j}, \quad T_{n} \overline{\overline{0}}_{0 \leq j \leq n} \tau_{j} .
$$

Assume $\left\{\epsilon_{n}\right\}$ is a sequence of numbers tending to zero such that $\epsilon_{n} \log n$ tends to infinity as $n$ tends to infinity, $\lim _{n \rightarrow \infty}\left(T_{n} / t_{n}\right)$ is also infinite, $\kappa_{n} / t_{n}=o\left(\epsilon_{n}^{-1 / 2}\right)$ and expectations of $a_{j}$ 's are zero. Then, outside an exceptional set of $P$, the number of solutions of $P(x)=K$ in the interval $(-1,1)$ is at least

$$
C \delta \epsilon_{n} \log n
$$

where $\delta=\Phi\left(-|K|-\frac{1}{2}\right)\left\{1-\Phi\left(-|K|+\frac{1}{2}\right)\right\}$. Also for $K=o\left\{\exp \left(n^{1 / 2-C^{\prime}}\right)\right\}$, the number of real solutions of $P(x)=K$ in $(-\infty,-1) \cup(1, \infty)$ is at least 


$$
C^{\prime \prime} \epsilon_{n} \log n
$$

The measure of exceptional set tends to zero as $n$ tends to infinity and, as usual, $\Phi(t)$ denotes the normal distribution function.

Theorem 2: Following the notation of Theorem 1, assume additionally, that all $\sigma_{j}$ and $\tau_{j}$ are finite and $K_{n}^{2}<n^{(1-7 \epsilon / 2)}(\log n)^{-\beta \epsilon}$ where $\beta>1$ and $\epsilon$ is any nonzero positive constant. Then, there exists an $\omega$-set whose measure is $O(1)$ for large $n$ such that, if $P_{n}(x)$ is not in this w-set, the number of solutions of $P(x)=K_{n}$ in the interval $(-1,1)$ is at most

$$
(C / \epsilon)\left\{\log \left(n / K^{2}\right)\right\}^{2}
$$

and in $(-\infty,-1) \cup(1, \infty)$ is

$$
C \log ^{2} n
$$

The measure of the exceptional set tends to zero as $n$ tends to infinity.

Theorem 3: If the coefficients of $P(x)$ are identically distributed with a common characteristic function $\exp \left(-C|t|^{\alpha}\right)$ with $\alpha \geq 1$, then outside an exceptional set there are at least

$$
C \log n /(\log \log n)
$$

number of real solutions of $P(x)=K_{n}$ for $\frac{K_{n}}{n} \rightarrow 0$ as $n \rightarrow \infty$. The measure of the exceptional set tends to zero as $n$ tends to infinity.

Theorem 4: If the coefficients of $P(x)$ satisfy the same assumptions as Theorem 3 and $K_{n}^{2}<n^{(\alpha-\alpha-\epsilon) / \alpha}(\log n)^{-2 \alpha \epsilon}$, then the results of Theorem 2 remains valid.

Theorems 1 and 2 show that in the interval $(-1,1)$ the number of solutions of $P_{n}(x)=K$ decreases as $K_{n}$ increases, while outside this interval there is no significant reduction at least up to $K_{n}=o\left\{\exp \left(n^{1 / 2-C}\right)\right\}$. Choosing constant $C$ small in Theorem 1 will allow the value of $K_{n}$ to be significantly larger than the upper limit previously found. This is also the case for Theorem 3 . However, because of nature of the distribution of coefficients there, the comparison with previous results is less natural. As far as the upper limit is concerned, by choosing $\epsilon$ small constant (independent of $n$ ) in Theorems 2 and 4 we can let $K_{n}$ be fairly close to $O(\sqrt{n})$, a case previously studied. The distribution of coefficients in Theorems 3 and 4 represent the asymptotic stable distribution with infinite variance (when $1 \leq \alpha<2$ ). For $\alpha=2$, the coefficients are normally distributed. 


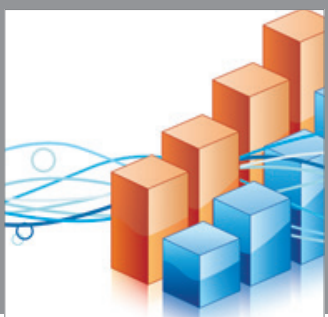

Advances in

Operations Research

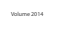

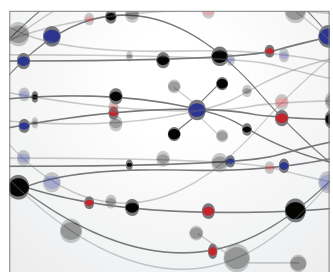

\section{The Scientific} World Journal
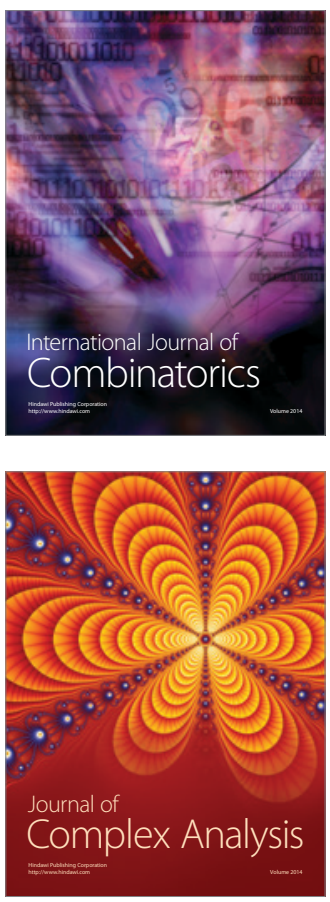

International Journal of

Mathematics and

Mathematical

Sciences
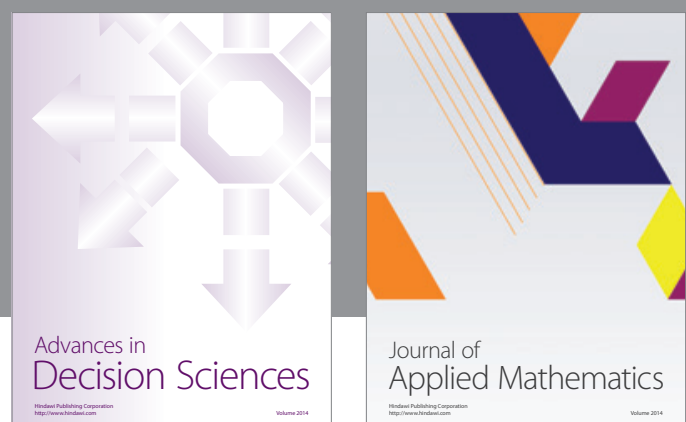

Journal of

Applied Mathematics
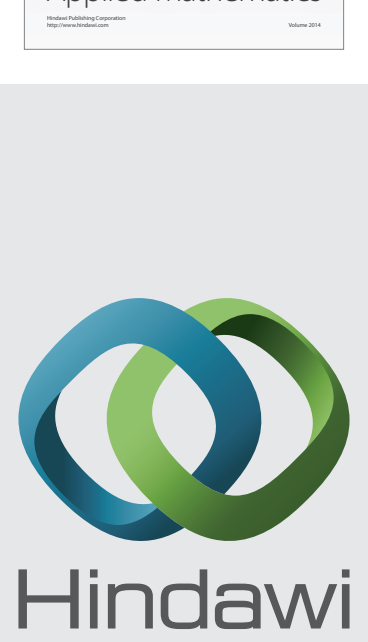

Submit your manuscripts at http://www.hindawi.com
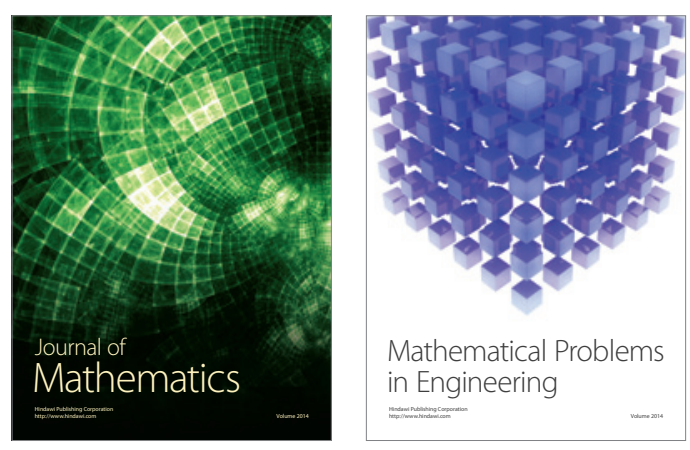

Mathematical Problems in Engineering
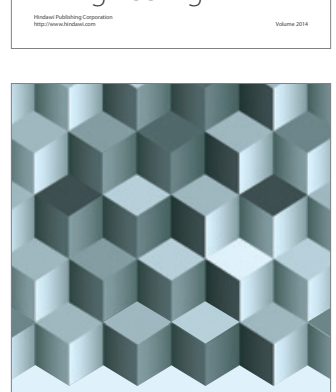

Journal of

Function Spaces
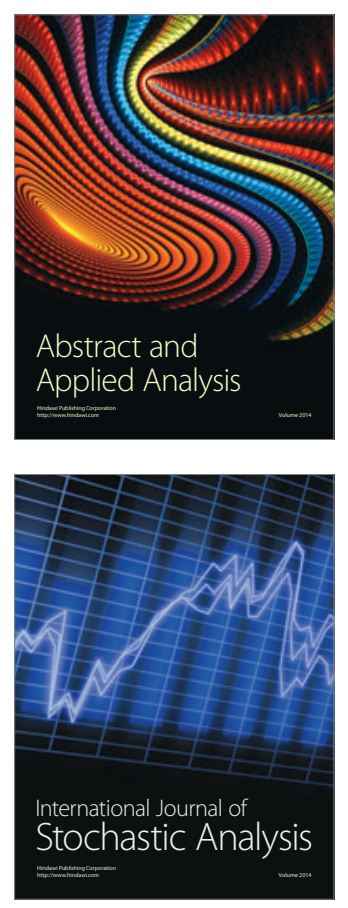

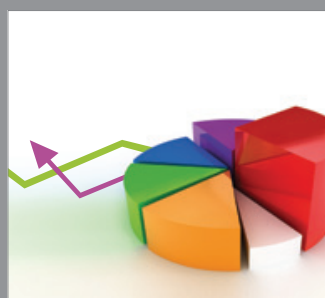

ournal of

Probability and Statistics

Promensencen
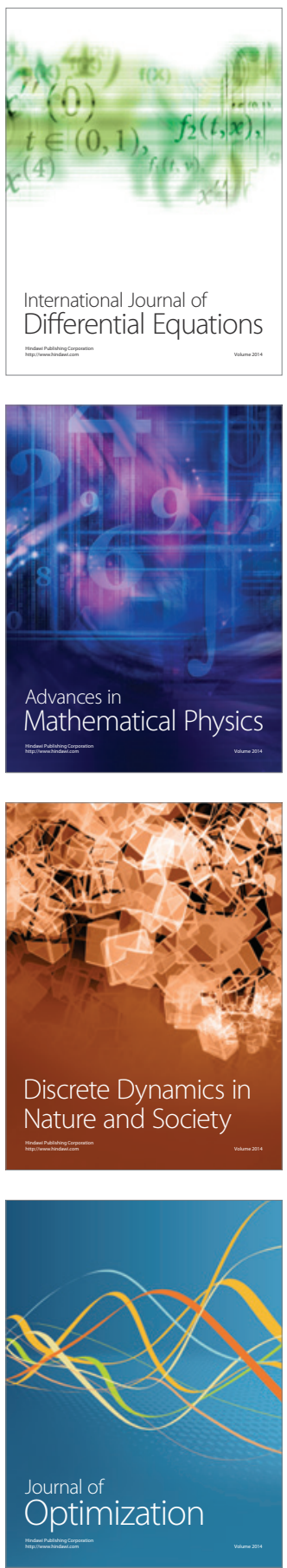